L'HOMME L'Homme

$183 \mid 2007$

Comment être parents?

\title{
Adopter des sœurs
}

Construction de la parenté et mémoire des origines

Françoise Zonabend

\section{CpenEdition \\ Journals}

Édition électronique

URL : http://journals.openedition.org/lhomme/25126

DOI : 10.4000/lhomme.25126

ISSN : 1953-8103

Éditeur

Éditions de l'EHESS

Édition imprimée

Date de publication : 1 septembre 2007

Pagination : 9-28

ISSN : 0439-4216

Référence électronique

Françoise Zonabend, « Adopter des sœurs », L'Homme [En ligne], 183 | 2007, mis en ligne le 01 janvier 2009, consulté le 01 mai 2019. URL : http://journals.openedition.org//homme/25126 ; DOI : 10.4000/ Ihomme.25126 


\section{Adopter des sœurs \\ Construction de la parenté et mémoire des origines}

Françoise Zonabend

LA PREMIÈRE CHOSE que l'on remarque en entrant dans la salle de ce pavillon accroché aux collines qui dominent la rade de Cherbourg (Manche, France) est une grande photographie traitée comme une gravure à l'ancienne, coloriée tel un pastel, encadrée et suspendue sur le mur du salon, au-dessus du piano. Elle représente trois fillettes à l'attitude posée, prises à mi-corps, assises de trois quart, serrées les unes contre les autres ; elles sourient et leur chevelure très noire ceint leur visage aux traits asiatiques. "Ce sont mes trois filles", dit mon interlocuteur, devinant mon interrogation ; "elles sont sœurs et coréennes" (cf. cliché infra).

Le charme de cette photographie de laquelle émane un calme heureux, le ton allègre et la tournure péremptoire utilisés par mon interlocuteur pour désigner les fillettes, le choc que me procura la vision de ces jeunes enfants aux origines lointaines venues vivre dans ce bout de France profonde où l'étranger était, il y a encore peu de temps, mal admis, le contraste entre ce que je connaissais par mes recherches ethnographiques sur le système traditionnel de parenté de cette région normande et ces nouvelles filiations revendiquées, m'incitèrent à poursuivre des investigations sur les changements intervenus dans l'univers social et parental de cette société locale, à partir de ce cas précisément. Certes, j'aurais pu élargir mes recherches auprès d'autres personnes qui avaient eu recours à l'adoption internationale, dans cette même aire géographique, collecter

Une première version de cet article a été présentée au colloque international «Perspectives contemporaines sur la parenté en Europe » organisé, à Paris, dans le cadre de l'École des hautes études en sciences sociales, par Marit Melhuus et Enric Porqueres i Gené, les 24 et 25 novembre 2005. 
d'autres histoires, d'autres récits, les comparer, les éclairer les uns par les autres ${ }^{1}$. Mais, en relatant cette histoire-là, dans toute sa minutie, il me semblait que je pouvais accéder à l'ensemble des éléments qui caractérisent notre système de parenté en général, tant dans ses dimensions verticales qu'horizontales, et dans son expression restreinte ou étendue, et que je pourrais mieux appréhender et analyser l'ensemble des relations familiales qui se tissent ou se rompent dans les groupes de parenté preneurs d'enfants, dès lors qu'on a recours à l'adoption lointaine pour "se fabriquer " des descendants. Enfin, située dans ce contexte normand, il était loisible de s'interroger sur la part que tient l'environnement local sur ces nouvelles façons de "faire famille »: les traits saillants du système local de parenté, le traitement dont ici l'étranger est l'objet, jouent-ils, d'une façon ou d'une autre, un rôle dans l'accueil de ces "parents » venus de loin?

\section{Parenté et adoption en Basse-Normandie}

Dans le Nord-Cotentin, la seule véritable parenté reconnue est la parenté consanguine, désignée par l'expression nos gens, c'est-à-dire l'ensemble des parents paternels et maternels qui appartiennent, dit-on, «à la même souche». Les alliés, qu'on appelle les biautés, sont traités, aujourd'hui encore, de "pièces rapportées". Au reste mari et femme ne sont jamais entre eux que des alliés, et la relation d'alliance unissant deux individus ne devient consanguine que pour la génération suivante, c'est-à-dire leurs enfants qui désignent père et mère sous le terme générique de mes gens (littéralement : ceux qui engendrent), expression où s'inscrit l'idée que le ou les enfants font le couple, mais où est connotée aussi la notion de proximité, de consanguinité à travers la procréation, entre parents et enfants, et entre germains. En sorte que, au cœur de la parentèle, constituée de l'ensemble des parents que l'on se reconnait, ressortent ces très proches consanguins. De fait, la ligne de démarcation entre les vrais parents et les autres passent ici entre mes gens, "mes père, mère, frères et sœurs non mariés ", et nos gens, "mes autres parents ». Dans cette perspective, on conçoit l'importance que revêt pour un homme et une femme mariés d'avoir des enfants, en nombre

1. Dans le département de la Manche, entre 1989 et 2003, les demandes d'agrément pour adoption ont triplé. En 2003, 136 dossiers ont été examinés, sur lesquels 81 agréments ont été accordés. Mais, à cette même date, 193 dossiers agréés étaient encore en attente d'enfants. Cette même année, 28 enfants français et étrangers ont été placés en vue d'adoption, dont 3 pupilles de l'État (nées sous X). La répartition des enfants par pays était la suivante: France 3; Rép. de Crimée 2; Haïti 1; Chine 1; Guatemala 2; Thaïlande 1; Philippines 1; Burkina Faso 1; Colombie 2; Éthiopie 5 ; Vietnam 1; Brésil 2; Bulgarie 1; Madagascar 5. Répartition par âge: 0 à 1 an : 10 ; 1 à 3 ans : $7 ; 3$ à 5 ans : $2 ; 5$ à 9 ans : 8 ; plus de 10 ans : 1. Les statistiques sont muettes sur les éventuels liens de parenté existants entre les adoptés (source: rapport d'activités, 2003, Service départemental d'Action Sociale, Caen). 
point trop important certes, mais suffisant pour assumer les rôles que cette cellule parentale assigne à chacun de ses membres : pérennité du lignage et continuité du groupe restreint.

La pérennité du lignage était jadis assurée à travers la transmission du patrimoine tant matériel qu'immatériel, distribué égalitairement entre tous les héritiers du couple. Il faut savoir, en effet, que ce noyau étroit formé par le couple conjugal et sa progéniture était et est encore assorti de formes d'héritage où chaque enfant, garçon comme fille, doit obligatoirement avoir part égale. Aussi, à chaque succession, l'ensemble des terres, des maisons, des jardins, des biens mobiliers est-il partagé en autant de lots égaux qu'il y a d'enfants. Ces pratiques successorales, auxquelles on est ici fortement attaché, fondent certes une égalité et une solidarité entre germains, mais, à terme, entraînent un effritement excessif des patrimoines fonciers. Il fallait donc, à chaque génération, avoir des descendants légitimes à qui transmettre les biens du lignage et, dans le même temps, veiller à ce que cet héritage ne s'émiette pas démesurément. Pour pallier ce dernier inconvénient, l'un des moyens possibles, à côté des mariages préférentiels entre cousins, consistait à restreindre le nombre de bénéficiaires en contrôlant les naissances ou, pour les germains, en surnombre ou non, à ne pas se marier.

Ainsi ceux qui, dans la fratrie, étaient célibataires demeuraient auprès des vieux parents, assurant la continuité de la cellule de base par la poursuite le plus longtemps possible de la vie en commun entre parents et enfants non mariés. Cette vision des choses était si fortement ancrée dans les mentalités qu'il était impensable, il y a peu de temps encore, de laisser un père et une mère vivre seuls, de sorte que, dans chaque fratrie, un enfant au moins restait célibataire pour s'occuper des vieux parents jusqu'à leur mort. S’il n'y avait qu'un enfant, tout naturellement il ne se mariait pas, car on préférait voir transmettre l'héritage à un proche cousin ou à un neveu, plutôt que de laisser vivre et mourir ses parents dans la solitude. Souvent deux germains, deux frères ou deux sœurs, ou un frère et une sœur, décidaient de ne pas se marier pour s'occuper de leurs vieux parents. Ces derniers disparus, les premiers demeuraient ensemble le restant de leur vie. On conçoit, dès lors, que le vécu en commun d'un destin semblable scellait, entre germains, une puissante solidarité (cf. Zonabend 1991).

Dans ce contexte de resserrement sur la parenté consanguine, de prééminence de la famille nucléaire, de cette force de la germanité, assortis du souci de transmettre le patrimoine familial au sein d'une filiation légitime, l'adoption d'enfant n'était guère favorisée. Aussi quand le malheur survenait dans un couple, ou que l'un ou l'autre des parents décédait, les orphelins étaient "ramassés ", c'est-à-dire qu'on se les répartissait au sein de la 
parenté proche. Mais, comme me l'a dit un informateur, "autrefois, on n'adoptait pas. On les prenait, ça oui! Mais même si on n'avait pas d'enfant soi-même, on ne les adoptait pas ".

Certes, aux couples stériles, aux célibataires sans descendance, on offrait couramment un neveu ou une nièce en parrainage, ou on les chargeait, plus volontiers, de s'occuper des orphelins de la famille; ils pouvaient alors les avantager quelque peu au niveau du patrimoine, mais jamais ces parents-là n'adoptaient officiellement leurs filleuls ou leurs neveux et nièces orphelins.

Autrefois, un homme, une femme ou un couple dépourvus de descendance, ne s'en fabriquaient pas une par le détour de l'adoption. Il est vrai que, dans ces temps-là, les seuls enfants susceptibles d'être adoptés - puisque les orphelins de famille n'étaient pas adoptables - étaient ceux qui avaient été placés, pour diverses raisons, dans un orphelinat. Enfants nés d'amours illégitimes ou ancillaires, enfants trouvés, ils furent pourtant nombreux dans la région si l'on en croit la rumeur locale et la littérature régionale et même nationale ${ }^{2}$. Mais ces orphelins, traités de façon péjorative de "bâtards" ou de "poussins de haie", étaient victimes d'un tel ostracisme social qu'il n'était guère facile - sauf subterfuge (Zonabend 2004) - de les faire admettre au sein d'une parenté légitime.

On peut rapprocher de ces façons de percevoir l'adoption, la méfiance dont on entourait le horsain - terme vernaculaire employé pour qualifier toute personne n'habitant pas la même commune et, surtout, pour désigner l'étranger, un individu venu d'une autre région ou, pis encore, d'un autre pays, et qui de ce fait était difficilement admis au sein du groupe local. Il est vrai que si cet étranger faisait "souche" dans le pays par mariage, il était peu à peu inséré dans la communauté villageoise et s'appropriait son identité locale.

Depuis quelques décennies, ces mœurs familiales et sociales se sont desserrées. Alliance et filiation ne sont plus aussi étroitement imbriquées. On choisit librement son conjoint. Les horsains, de plus en plus nombreux dans la région, sont mieux admis, même en dehors de tout attachement familial local. Les enfants de paysans quittent la terre qui n'est plus un enjeu patrimonial et lignager. La société se diversifie et s'ouvre sur l'extérieur, les enfants ne restent plus auprès de leurs vieux parents. Les femmes ont accès à la contraception ou à l'interruption volontaire de grossesse, dès lors les enfants procréés hors mariage, presque toujours reconnus par leur père, compagnon de la mère, ne sont plus l'objet d'opprobre. On ne parle plus de "bâtard » ni de " poussin de haies "; au demeurant, ils ont disparu.

2. On se reportera, à ce sujet, à quelques nouvelles de Guy de Maupassant ou de Jules Barbey d'Aurevilly dont l'action se situe dans cette région normande. Quant aux récits régionaux, on lira le tout dernier livre paru sur ce thème de Jacques Rouil (2005). 
Les couples cherchent avant tout leur épanouissement personnel, sans plus penser à l'enrichissement patrimonial de la famille et, s'ils sont inféconds et désirent avoir des enfants, ils ont recours à la procréation médicalement assistée ou, en cas d'échec, à l'adoption, aujourd'hui essentiellement internationale. Comment, dès lors, se fabriquent ces nouvelles formes de filiation? Sont-elles marquées par la culture locale et la forme du système de parenté normand? Cette forte consanguinité, cette solidarité entre germains, repérées dans les familles de jadis, pèsent-elles encore sur la fabrication des parentés actuelles? La propension à différencier les personnes en fonction de leurs origines géographiques ou culturelles joue-t-elle toujours un rôle? Peut-on tenter de repérer les effets de ces traits à partir du cas de l'adoption de nos trois sœurs d'origine coréenne?

\title{
La fabrication d'une filiation
}

Georges et Maryvonne D. sont tous deux originaires de la même commune du canton de Beaumont-Hague, dans le département de la Manche. Ils se sont mariés en 1972, lui était employé de banque, elle, travailleuse sociale. Ils sont aujourd'hui tous deux retraités. Ils n'ont jamais pu avoir d'enfant malgré les nombreux traitements médicaux suivis avec opiniâtreté pendant plusieurs années. Ils décidèrent donc d'adopter un enfant et se tournèrent vers l'adoption internationale. Par l'intermédiaire de la DDASS, ils s'inscrivirent à une association spécialisée dans ce genre de demande qui était alors en contact avec la Corée. Les démarches durèrent cinq ans. Un premier dossier - un garçon - leur fut proposé ; ils acceptèrent, mais, à la dernière minute, cela échoua. Très déçus, ils pensèrent alors qu'ils étaient trop âgés et qu'on ne leur confierait pas d'enfant. En 1982, un jour enfin, Mme D. reçut un coup de téléphone à son travail :

\begin{abstract}
"On a trois filles, trois sœurs à vous proposer. Les voulez-vous? " Elle appelle son mari à son bureau, celui-ci répond aussitôt : "Pourquoi pas, qu'on nous envoie le dossier... » Et de m'expliquer alors: "Tant que vous n'avez pas le dossier avec les photos, les papiers de santé, de scolarité, tout ça... Alors une fois que vous avez eu ça... Les enfants sont à vous".
\end{abstract}

L'adoption est bien une parenté "choisie " (Fine 1998), mais les raisons de ce choix «sur dossier» restent, pour les parents adoptants, assez obscures $^{3}$. Les photographies des enfants, leurs résultats scolaires ou leurs bulletins de santé joints au dossier, sont les indices sur lesquels ils se basent pour assurer le bien-fondé de leur adhésion. La photographie et les papiers

3. Lorsque le choix de l'enfant à adopter se faisait directement, comme au Québec par «magasinage » dans un orphelinat, les parents pouvaient arguer que les enfants, d'un regard ou d'un geste tendre, les avaient choisis (Collard 1996). 
officiels d'identification des enfants jouent donc un rôle médiateur important dans le processus d'appropriation des enfants par les parents adoptifs... C'est à ce moment-là, au vu des portraits qui, pour eux, font signes, de descriptions écrites qu'ils déchiffrent avec leur sensibilité, que s'enclenchent les liens affectifs et s'engage le processus de parentalité. "Le dossier ", avec photos et papiers, est le point de départ, la marque tangible de l'étape qui va mener l'adopté au sein de sa nouvelle famille, tout comme la radiographie de la première échographie scande, pour le couple de parents, l'arrivée du bébé parmi eux (Belleau 2000). Pour tous, ces documents "princeps " sont empreints de la même émotion parce que se révèlent ici l'altérité et l'identification nécessaires pour s'intégrer au groupe.

La photographie tient d'ailleurs une place prépondérante dans le processus d'intégration des fillettes adoptées au sein de la famille D. Le jour même de leur arrivée à la maison, tout comme à chaque moment ordinaire ou extraordinaire de leur nouvelle vie normande, des clichés ont été pris en guise de commémoration ou de remémoration de ces premiers temps de vie en commun. Toutes ces photographies sont encadrées, entassées les unes auprès des autres, et disposées sur les murs, les meubles de toutes les pièces du pavillon, de la cuisine au salon. La plus solennelle est, nous l'avons dit, suspendue en évidence dans la pièce de réception. Ces multiples photographies, affichées partout, ont sans aucun doute permis au couple adoptant de mieux s'identifier, de s'incorporer ces enfants culturellement et physiquement différents. Par la captation intense de l'image, son étalage surabondant dans l'espace domestique, ils s'efforcent de faire de ces enfants des membres à part entière de la famille. Au demeurant ces processus de signalement par photographies interposées, accompagnés d'un changement de noms, du port de vêtements nouveaux, participent, à n'en pas douter, à l'appropriation par intégration des enfants dans le groupe familial. Mais, dans le même temps, cet empressement à graver sur la pellicule ces moments de (re)connaissance, leur accrochage profus et visible dans la maison d'accueil, constituent, pour les adoptées, un rappel incessant de la place qui leur est désormais dévolue et à laquelle elles doivent se tenir au sein de la parenté adoptante. Cette mise en scène iconique, ces attouchements, accoutrements, modifications anthroponymiques, s'apparentent aux rituels d'accueil que nombre de sociétés ont mis en place pour assimiler l'Autre - étranger ou ennemi potentiel - à Soi ${ }^{4}$.

4. Cf. à ce propos Luc de Heusch (2002). On trouvera sur ce point des rituels d'appropriation et d'intégration, des réflexions importantes, même si elles portent sur un tout autre sujet, dans l'article de Alain Testart (1999), et par le même auteur, et dans L'Homme (1999: 191-193), son compte rendu de l'ouvrage de Roland Viau, Enfants du néant et mangeurs d'âmes : guerre, culture et société en Iroquoisie ancienne, Québec, Éd. du Boréal, 1997. 
Lors de son arrivée, l'enfant adopté est encore situé entre deux familles, entre deux parentés ; il n'appartient plus à l'une mais n'est pas encore intégré à l'autre. Il est, en quelque sorte, en marge et subit donc, comme toute personne associée à cet état frontalier et somme toute dangereux, les regards curieux, mais aussi méfiants et soupçonneux de tous ceux qui entourent la nouvelle famille. Les photographies qui captent son image, exposent son altérité, sont étalées au vu et su de tous et agissent comme autant de signes d'appartenance au groupe, au lieu, au milieu. Elles participent de son intégration sociale, tout comme le prénom et les vêtements "occidentaux" dont il est rapidement pourvu le transforment en apparenté.

On comprend dès lors que dans cet étalage profus d'images placées en évidence dans la maison, aucune ne date "d'avant ", du temps de la Corée, ni ne rappelle ce pays. Pourtant les fillettes sont arrivées avec tout un lot de photographies illustrant leur vie là-bas, mais celles-ci ne figurent pas dans cette (dé)monstration. Leur nouvelle vie, telle une nouvelle naissance, commence là, au moment où elles entrent dans la maison, et tout est mis en place pour rappeler, aux enfants comme aux autres, cette nouvelle naissance, celle-ci symbolique.

«Elles sont arrivées à la maison, le 16 juillet... », se souvient leur père. «Tiens la photo qu’est dans la cuisine a été prise ce jour-là ».

Pour autant « leur vie d'avant " ne leur est ni cachée ni interdite de parole. Les fillettes, âgées de dix ans et demi, de sept et cinq ans, lors de leur venue en France, savent tout de leur origine biologique, et elles ont même renoué, nous le verrons plus loin, avec leur famille natale. Mais de cela, elles ne veulent pas parler avec leurs parents adoptifs ni partager avec eux quoi que se soit de leur vie antérieure.

«On a eu des livres en coréen pour elles, elles n'en voulaient pas. On a voulu apprendre la cuisine coréenne, elles ne voulaient pas. Leurs photos de Corée, c'est à elles, on les a jamais regardées ensemble, elles les gardent. C'est leur monde, on n'en parle pas ", commente leur mère adoptive.

Comme il est fréquent dans les familles adoptives, Monsieur et Madame D. ont tenté de substituer à un savoir et une recherche sur les origines biologiques des fillettes, des connaissances sur leur pays natal. Était-ce pour posséder avec elles une mémoire commune? Les filles ne furent guère coopératives dans cet effort d'acquisition de leur propre culture. Elles refusèrent tout simplement de s'y intéresser : jamais elles n'acceptèrent de partager leur savoir, leur mémoire ou leurs souvenirs, pas plus qu'elles ne voulurent nouer des liens amicaux avec d'autres jeunes Coréens adoptés par des familles voisines et qui leurs furent présentés lors de réceptions ou de fêtes données à cet effet. Leur intégration à leur nouvel univers social 
et parental passait par une totale assimilation au groupe d'accueil sans que celui-ci n'eût rien à connaître de ce qu'était leur monde à elles.

En d'autres termes, le monde de leurs origines a fonctionné comme une catégorie opérant une sélection entre des supports d'incompatibilité dans le domaine de la communication. Leur monde de là-bas était leur domaine, leur bien personnel, et aucune personne d'ici ne devait transgresser ce mode de penser, de se comporter. À cela s'est ajoutée, à n'en pas douter, la force de leur sororité pour entretenir entre elles, et pour elles seules, leur mémoire parentale et culturelle natale. Elles n'acceptèrent aucun support mémoriel extérieur. Mais les fillettes eurent la chance d'emporter avec elles et de détenir en partage, tel un viatique, tous ces papiers mémorables et ces souvenirs remémorés.

Tout s'est passé comme si leur vie "d'avant " leur appartenait en propre. Elles sont restées les gardiennes et les gestionnaires de la mise en forme narrative, émotionnelle et mémorielle de leur patrimoine natal. Entre ces deux mondes - le natal et l'adoptif -, il y eut coexistence pacifique des sources de la parenté et de la culture. Elles connaissaient et manipulaient, sans doute par l'intermédiaire de l'aînée, l'histoire de leur filiation biologique. Il n'y eut pas choc de deux vérités filiatives, mais superposition et acceptation, de part et d'autre, de ces deux mémoires, l'une passée et de là-bas, l'autre à construire ici (Collard, à paraître).

Effectivement, l'intégration familiale et identitaire des trois sœurs s'est faite en séparant le monde "d'avant", celui qu'elles ont connu jusqu’à leur venue en France et celui "d'après" qui les a accueillies et adoptées. Entre le monde d'ici et celui de là-bas, on ne s'ignore certes pas, mais on ne communique pas.

"À l'âge où on les a eues, il n'était pas question de leur cacher leur origine, mais on n'en parle pas. Ici, elles sont normandes.»

Il est important de noter la forte insistance - nous y reviendrons - mise par les parents adoptifs à intégrer les trois sœurs dans leur propre univers affectif et social, sans s'immiscer dans le leur. Cependant - nous l'avons dit - les relations des filles avec la Corée et surtout avec leur famille d'origine n'ont jamais été rompues. Monsieur D. me raconte :

"La mère [des fillettes] a dû demander notre adresse à l'orphelinat... Une de ses sœurs, qui vit aux États-Unis, a téléphoné un jour à la maison; elle parlait français; elle nous a demandé comment allaient les filles... Puis ce fut la mère qui parfois téléphonait ou envoyait une carte aux filles. Enfin, la mère a dû avoir des nouvelles par des étudiants coréens qui étaient à l'université de Caen et ont rencontré nos filles qui y étudiaient... C'est par eux que la mère a su qu'une des filles venait en Corée, elle l'a trouvée et rencontrée... Je sais qu'elle a mangé chez sa mère, je ne sais pas si elle y a vécu. On n’en a pas parlé... » 


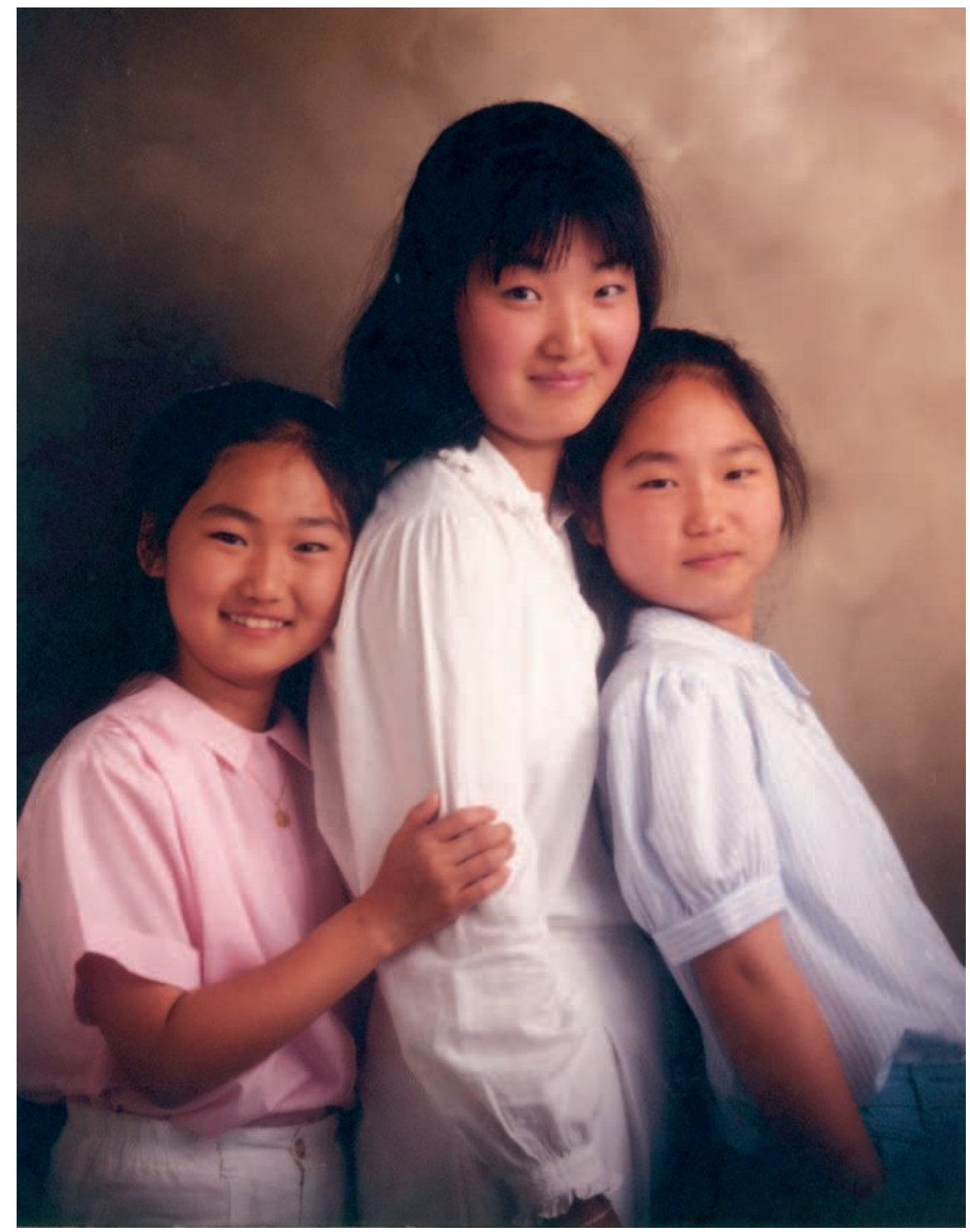



La mère biologique envoie donc, encore aujourd'hui, une carte ou un mot chaque année pour leur anniversaire, et même parfois elle téléphone. Jamais les parents adoptifs n'ont demandé à savoir ce qu'elles se disaient, jamais les filles n'ont relaté ce qu'elles s'étaient raconté. Puis, il y eut le voyage d'une des filles en Corée. C'est l'aînée qui, à l'université, a voulu se perfectionner dans sa langue natale qu'elle parlait encore, et elle eut l'occasion, lors d'un stage linguistique financé par le département universitaire, de retourner en Corée. À cette occasion, elle y a donc revu sa mère.

"Mais on en a peu parlé, j'crois que ça s'est mal passé "s, m’affirme Madame D. qui ne sait pas, vraiment, si la jeune fille a rencontré d'autres parents paternels ou maternels. «J'crois qu'elle a revu des tantes... Mais de quel côté? J'sais pas trop sur le père, il a été très malade, est resté longtemps à l'hôpital où il est mort, il a fallu payer, c'est pourquoi elles ont été mise à l'orphelinat... »

Les cadettes, quant à elles, ont oublié le coréen et n'ont pas voulu le réapprendre (la seconde a refusé d'aller en Corée, alors qu'elle aurait pu s'y rendre durant son voyage de noces). Pour elles, la rupture est accomplie. Les relations, qui existent toujours avec la mère biologique, se tissent donc uniquement par l'entremise de la sœur aînée qui joue, auprès des cadettes, le rôle de passeur et de transmetteur de l'histoire de leurs origines. Le groupe de germains trouve, par ce biais, sa solidarité et son homogénéité, son assise originelle commune sur laquelle il peut s'appuyer face à la parenté adoptive. Il forme un bloc soudé, uni parce qu'apparenté et solidaire ${ }^{6}$, avec une histoire familiale, une mémoire généalogique originelle qui n'appartiennent qu'à lui seul, et qu'il n'entend partager avec quiconque. Cette façon d'agir, en groupe de germains, a sans doute donné, à ces sœurs, une grande force pour s'ouvrir sur une autre parenté et s'identifier, sans trop de souffrances, à leur nouvel univers culturel, familial et affectif. Surtout qu'adoptées ensemble par un couple conjugal, leur germanité biologique s'est trouvée renforcée par une germanité sociale.

On remarquera, afin de bien situer la façon dont s'est jouée ici la construction identitaire de ces adoptées avec toute la charge relationnelle qu'elle sous-tend, que ce ne sont pas les parents adoptifs qui ont payé, à la

5. Il semble, d'après ce qui était noté dans le dossier remis par l'association, que les fillettes aient été mises en orphelinat par leur mère devenue veuve, pour être adoptées. Si celle-ci avait gardé ses enfants, elle n'aurait pu trouver à se remarier. Actuellement, la mère est remariée et elle a un fils. Elle aurait demandé à sa fille, lors de sa venue en Corée, si les parents de France ne voulaient pas prendre cet enfant pour qu'il soit mieux éduqué... Cette demande a beaucoup choqué la jeune fille qui a dit, en guise de simple commentaire, "elle vous croit riches». Cette situation résume bien toute l'ambiguïté que recèle l'adoption internationale : du côté des donneurs on espère toujours, malgré l'adoption plénière, tirer quelque avantage de la situation (Collard 2005).

6. L'aînée et la benjamine sont marraines des enfants de la seconde. La sœur aînée, ainsi que la mère adoptive naturellement, était auprès de la puînée lors de ses accouchements. 
jeune fille, ce voyage au pays natal. Sur ce point, personne, ni adopté ni adoptant, n'est en dette et les deux mondes, celui des origines et celui d'accueil, demeurent séparés. Il en fut d'ailleurs de même lors de l'arrivée des fillettes en France. Les parents ne se sont pas rendus en Corée chercher les enfants. Ils ont payé les voyages de ces dernières jusqu'à Paris après avoir acquitté leur dû à l'orphelinat par l'intermédiaire de l'association. Pour payer cette somme, ils ont été aidés par la caisse d'assurance familiale du département et par une collecte faite par le comité d'entreprise de l'établissement où travaillait Monsieur D. Le cercle de solidarité s'est donc élargi et tout le poids financier de l'adoption n'a pas pesé que sur les seuls parents. Cette prise en charge collective du coût de l'adoption a abouti, à n'en pas douter, d'un côté comme de l'autre, à diluer l'idée de dette. Les adoptants n'ont pas eu à fournir un effort financier démesuré pour posséder les enfants ; ils n'ont surtout pas eu l'impression de faire à ces derniers un don dont ceux-ci seraient à jamais débiteurs ${ }^{7}$.

Tout en continuant ainsi à avoir accès à leur propre histoire familiale, les fillettes ont été rapidement intégrées dans l'univers parental des adoptants. Au début, il y eut bien quelques réticences. Ainsi, la mère du père adoptif a-t-elle bougonné : "Oh ! J'sais pas si ça va être pareil avec ces nouvelles filles venues de loin...» Mais très vite elle a été conquise par les fillettes et ne fait plus de différence avec ses autres petits-enfants, aussi estce à la cadette des filles qu'elle a récemment donné sa précieuse machine à coudre «mécanique ». Les adoptées sont devenues " héritières » au même titre que les autres petits-enfants.

L'intégration au groupe familial s'est faite d'abord à travers l'usage des appellations de parenté que les fillettes employèrent d'emblée pour désigner les membres de la parentèle adoptante. Les neveux et nièces sont, pour elles, des " $\operatorname{cousin}(\mathrm{e}) s$ »; pour eux, elles sont les « cousines chinoises ", sans qu'aucune péjoration ne se glisse dans cette caractérisation. Les oncles et tantes sont "tata" ou "tonton", et une belle-sœur qui avait refusé d'être dénommée ainsi a été bannie des relations du couple adoptant. Le non-acquiescement au geste de l'adoption est considéré comme une insulte. Il entraîne la rupture des relations familiales. C'est comme si les enfants avaient été traités de bâtards et les parents d'irresponsables. Certes, jadis, nous l'avons dit, on n'admettait pas que n'importe quel inconnu, un étranger, entrât dans l'univers familial. Mais si un parent choisissait un horsain pour faire partie de sa famille, alors celui-ci devait y être accueilli. Il en va de même aujourd'hui, et toute autre attitude ne saurait être tolérée. 
Toutefois, Monsieur D. prend bien soin de me préciser :

«J'ai bien vu sur les photos qu'elles étaient d'une très bonne famille... J'ai su aussi qu'elles sont de Séoul, c'est la Corée du Sud, elles sont de race très pure... qu’on m’a dit..."

Les adoptants cherchent eux aussi à en apprendre un peu plus sur les enfants qu'ils accueillent. La quête des origines taraude aussi le père et la mère adoptifs, et ils ne sont pas peu fiers de pourvoir faire état de telles références, ici, en Normandie - une région encore toute embrumée par la conquête viking et sensible au poids symbolique qu'impose cette "race " dite civilisatrice qui rayonne, dit-on encore aujourd'hui, sur le pays (Lechanteur 1974). La parenté adoptive a besoin, comme la parenté par le sang, de se rassurer sur les origines biologiques des enfants, tout comme ces derniers ressentent, à un moment ou à un autre, le besoin de savoir d'où ils viennent (Sagnes 2000). Ainsi, culturellement et ethniquement intégrées, les fillettes ont-elles été aussi initiées à l'univers parental des adoptants. Cette initiation s'est faite tout naturellement lors des réunions familiales ou, plus systématiquement, par le commentaire des photographies de famille des adoptants.

"Sur chaque photo, il a fallu que je leur énumère tous les parents qui étaient là et le lien de parenté que j'avais avec eux ", raconte le père.

Participant de la sorte au groupe familial, les fillettes ont acquis et incorporé le savoir généalogique qui se transmet traditionnellement au sein des parentèles normandes. Leur irruption au sein des parents des adoptants n'a entraîné ni rupture ni hiatus. Remarquons d'ailleurs que Monsieur D. a trois frères et sœurs qui ont chacun trois enfants. Les trois sœurs viennent donc tout naturellement s'insérer dans cet ensemble fraternel, formant elles aussi bloc, fortes de leur sororité qui leur confere un statut biologique, les posant d'emblée à égalité avec toutes ces personnes parentes par alliance ou par consanguinité. Face à cette parentèle, elles n'étaient pas dépourvues de tout état parental et pouvaient même passer sous silence tout ce qui avait trait à leur propre ascendance. Nous y reviendrons plus loin.

\section{Paternité, maternité, germanité}

Toutefois, c'est au sein du groupe nucléaire des parents adoptifs que tout s'est joué, que la filiation s'est véritablement fabriquée, consolidée au fil du temps. Du côté des fillettes comme de celui des parents, l'appropriation des uns par les autres s'est déroulée selon un long processus d'intégration ponctué à chaque étape, de moments ou d'événements, qui l'assimile à un véritable roman familial. Énumérons quelques faits ou traits qui émaillent cette histoire. 
À partir de l'acceptation du dossier, se produit pour le père et la mère un processus de "biologisation" des liens filiaux qu'ils ont noués. Monsieur D. raconte que, lorsqu'il a su que l'adoption était acceptée par les diverses institutions de tutelle, celle-ci allait enfin aboutir :

"J'ai été malade comme un chien pendant deux jours ». À cette réflexion répond celle de sa femme: «Moi, j'étais euphorique».

Mari et femme réagissent à l'annonce de la réussite de l'adoption comme un couple qui apprend qu'il attend un enfant; ils ressentent les mêmes symptômes de décompensation physique et/ou psychologique que connaissent les futurs pères et mères. Et, dès lors, le temps de l'adoption est vécu, pour l'un comme pour l'autre, comme une véritable grossesse. Le couple s'est trouvé pris, physiquement et affectivement, dans un véritable processus de gestation symbolique (Delaisi de Parseval 1981).

Puis, il y eut le moment de l'arrivée que l'on peut assimiler à une naissance. Monsieur et Madame D. sont allés accueillir les fillettes à l'aéroport à Paris, et d'insister :

"C'est là qu'on les a eues, qu'on les a senties à nous.»

Au demeurant, c'est à cet instant que débute la (re)connaissance réciproque des adoptés et des adoptants. Ne partageant aucun langage en commun, chacun a dû apprendre à faire connaissance avec l'autre.

«On a communiqué comme si c'étaient des bébés. On ne parlait pas la même langue, alors on montrait, on faisait des gestes. Oh ! ça m’a manqué », insiste le père adoptif, «de ne pouvoir leur parler, leur demander ce qui leur était arrivé, leur histoire... On a tant envie de savoir. Mais c'était peut-être mieux ainsi. »

Ce silence obligé, cette phase de latence imposée, cette période de marge vécue comme un commencement ont peut être permis que se trame entre les protagonistes un tissu relationnel harmonieux où chaque mémoire familiale a trouvé sa place. En ces circonstances de mise et de prise de connaissance réciproque, il faut laisser du temps au temps.

Peu après leur installation, la plus jeune des fillettes, âgée de cinq ans, demanda à être allaitée par sa mère adoptive. Celle-ci s'exécuta et donna, virtuellement bien sûr, son sein à sucer à l'enfant pendant près de deux ans. La mère ne s'étonna pas de cette demande, on l'avait prévenue que de jeunes adoptés étaient susceptibles de régresser lors de la survenue de tels événements. Ce simulacre d'allaitement de la petite dernière vient renforcer, par agrégation sororale, les liens maternels qui se tissent entre la mère et les autres sœurs. À cette initiation et identification de la mère adoptive à la maternité répond le récit masculin de fabrication de la paternité : 
«Un jour une des filles m’a tellement embêté que je lui ai donné une bonne fessée. Elle a pleuré, puis elle est revenue. On était beaucoup mieux après. Un ami m'avait dit "Tu verras, se sera vraiment ta fille quand tu lui auras donné une fessée...” Et c'est vrai !»

Du côté des fillettes, le même processus d'apprentissage d'une autre mère, d'un autre père, doit aussi se mettre en place. Sans doute l'aînée qui possédait l'histoire et la mémoire de leur filiation natale a-t-elle pris une place décisive dans ce vécu identitaire.

"L'aînée a toujours joué son rôle. Elle les surveillait et les réprimandait si elles ne se comportaient pas bien avec nous. Elle leur donnait un grand coup sur la tête... Fallait voir!»

Tout en entretenant une relation réelle avec sa génitrice et sa famille d'origine, l'aînée a pu et su opter pour sa parenté adoptive et transmettre ou imposer cette volonté à ses sœurs, mais aussi la manifester auprès de ses nouveaux parents.

«Un jour, comme ça, l'aînée m’a donné la montre qu'elle portait au poignet en arrivant. C'était une montre d'homme, une Seïko à quartz... Était-ce la montre de son père? Je ne sais pas, je ne lui ai jamais demandé, mais je la porte toujours ", raconte Monsieur D.

L'aînée a donc, par son don, scellé le lien paternel tout comme la plus jeune, en suçant le sein de sa mère adoptive, a fabriqué de la maternité.

Enfin, les mécanismes de dénomination et d'appellation dont tous vont bénéficier, ont joué leur rôle intégrateur.

"Dès le premier jour, en France, elles nous ont appelés "opa” et "oma” et on est toujours restés comme ça " [remarquons que la mère biologique est désignée, en français par les filles, sous l'appellation de «la mère de Corée »].

Quant aux fillettes qui usaient et usent encore parfois entre elles d'un appellatif coréen signifiant "petite ou grande sœur" ou "sœur cadette", elles refusent que leurs nouveaux parents les dénomment ainsi. Elles reçoivent alors des prénoms français, officialisés juridiquement lors de l'adoption plénière et, rituellement, au moment du baptême chrétien. Chaque protagoniste incorpore donc l'autre dans son propre système culturel, révélant ainsi comment ces appellatifs ont pu jouer, pour chacune des parties, un rôle d'assimilation et d'harmonisation réciproques : chacun a fait un pas vers l'autre.

Bien que les fillettes ne sachent pas un mot de français, deux mois après leur arrivée, les parents adoptifs les ont mises à l'école communale du village, dans des classes correspondant à leur âge réel. Elles ont rapidement surmonté leur handicap et réussi à suivre une scolarité normale. 
«Elles ont tout fait comme les autres. Le 11 novembre, le monument aux morts, le catéchisme...Tout quoi...»

Entre elles, les fillettes ont continué à parler coréen pendant un certain temps, puis le français a, peu à peu, remplacé leur langue de communication intime. Aujourd'hui, elles se disent "françaises ", et ne veulent parler de leur histoire personnelle avec qui que ce soit, surtout pas avec l'ethnologue. Elles s'irritent qu’à la vue de leurs traits "exotiques », on s'étonne de leur patronyme à consonance française et qu'on leur demande d'où elles viennent. Désormais, elles se sentent si parfaitement intégrées qu'elles ne veulent pas avoir à s'expliquer sur leurs origines biologiques ni sur leur histoire de vie. De fait, elles perçoivent comme une insulte grave toute allusion à leur condition natale. Comme si, ce faisant, on leur rappelait leur position d'exclues de leur pays d'origine et leur statut de migrantes dans la société adoptive.

Cette façon de réagir se conçoit d'autant mieux qu'il faut comprendre que, de leur histoire adoptive, elles veulent surtout retenir l'aspect tout à la fois prodigieux, festif et affectif.

En effet, il faut insister, me semble-t-il, sur le fait que les parents et les enfants sont revenus sans cesse, des années durant, sur le récit de l'adoption. Ni les uns ni les autres ne se lassèrent jamais de l'entendre et celui-ci a agi comme une véritable saga des origines.

«Elles nous demandaient souvent comment, pourquoi on les avait choisies. Ce qui les étonnait, c'est qu'on les ait prises toutes les trois. Elles s'attendaient à être séparées et étaient émerveillées d'être ensemble. Alors, il fallait qu'on raconte l'histoire du dossier, qu'on explique comment et pourquoi on s'étaient décidés... encore et encore...»

À cette reconnaissance immense qu'éprouvent les jeunes filles envers leurs parents adoptifs d'avoir préservé le groupe sororal, répond la gratitude de ces derniers de se voir ainsi entourés et confortés dans leur statut conjugal, puisqu'en Normandie, l'enfant fonde le couple. Le récit de cette adoption constitue, entre les parents et les enfants, comme une sorte de "roman de la filiation" pour reprendre une expression de Freud. Chaque partie trouve matière à fonder, de façon peut-être magnifiée, l'histoire de leurs parentés (re)construites.

Aussi, entre les uns et les autres, il n'est pas question de dettes, nous l'avons déjà souligné, mais plutôt d'échanges où chaque récipiendaire, à égalité, a reçu son dû. Chacun, ici, s'inscrit dans le circuit sans fin de dons et contre-dons qui caractérise un groupe familial. Une telle relation, bien pondérée, permet alors que la plaisanterie s'installe. Ainsi les filles insistent-elles parfois sur le fait «qu'elles, elles n'ont pas choisi et qu'elles 
auraient pu tomber sur des parents plus jeunes, plus modernes..."; ceuxci répondent sur le même ton plaisant " qu'ils auraient pu avoir des enfants plus sages...» De cette filiation fabriquée, chacun peut plaisanter car personne n'est débiteur. Les discussions entre adoptés et adoptants ont pris la tournure de relations à plaisanterie qui désamorcent l'hostilité plus ou moins inscrite dans des déclarations péremptoires qui interviennent souvent à l'adolescence entre parents et enfants adoptifs. La « revanche adoptive", si souvent notée et médiatisée, ne semble pas s'être produite ici (Pisier 2004; Monestier 2005). Sont-ce les circonstances économiques et sociologiques dans lesquelles cette adoption s'est déroulée ou la force de cohésion du groupe sororal qui ont joué un rôle temporisateur?

Dans cette adoption, les processus assimilateurs sont rapidement en place et vont si bien fonctionner qu'aujourd'hui chaque protagoniste $\mathrm{y}$ trouve son compte: la (les) fille(s) imite(nt) leur père ("C'est à cause de toi si j'ai fait ce type d'études "); quant aux parents ils s'identifient à elles de façon quelque peu narcissique : «la cadette a la même voix que sa mère, je les confonds toujours au téléphone..."

Cette adoption s'inscrit désormais sur trois générations. L'aînée et la cadette vivent en concubinage avec des compagnons français d'origine. La première vient d'acheter une maison tout près de chez ses parents adoptifs : "Elle aime la région et veut rester par ici!», précise Monsieur D. La seconde est mariée avec un Français d'origine, et a deux jeunes enfants. L'on constate dès lors que l'existence de la lignée est assurée et que la cohésion parentale s'est effectuée :

"Pour les enfants de L., on est "papi" et "mamie". Pour l'instant ils ne savent rien de l'histoire de leur mère. Je ne sais si elle leur dira un jour".

Pour ces jeunes enfants, inscrits dans une large famille paternelle et maternelle, avec grands-parents, oncles et tantes, cousins et petits cousins, les solidarités intergénérationnelles et collatérales sont en place. Le passage a l'ancestralité s'est bien effectué.

\section{Filiation versus germanité ?}

Faut-il penser qu'ici la germanité a " poussé » la filiation, c'est-à-dire a aidé à son arrimage ? Déjà constitué « en parents » dans la fratrie, les fillettes ont pu donner sens, malgré les tribulations filiatives dont elles ont été l'objet, à la question: "Qu'est-ce qu'un parent?" Elles possédaient un socle dur, tangible, sur lequel s'appuyer, un langage signifiant prêt à être employé. L'univers de la signification de la parenté était présent. Quand toutes les places vacillent, quand les repères familiaux se dissolvent, il leur restait cela, 
leur "germanité» en partage. Quand les familles se recomposent, par divorce ou par adoption, les liens de parenté horizontaux sont tout aussi importants à évaluer que les liens verticaux. Le cercle de famille peut se réassurer, se recomposer autour de cette germanité qui agit, ici, comme une sorte de contrepoids à la forte et large inscription parentale du couple adoptant.

"La parenté d'origine sert donc d'opérateur pour reconstituer une base biologique commune dans les familles adoptives, ce qui leur donne ainsi plus de légitimité en tant que famille partiellement consanguine - ce critère restant toujours important dans les représentations occidentales " (Collard 2005 : 215-216) ${ }^{8}$.

Le processus adoptif a-t-il ici été d'autant plus facilité qu'il s'agissait d'une fratrie de même sexe ? Dans cette entité sororale, l'aînée a joué, nous l'avons observé, auprès des cadettes un rôle de passeur mémoriel. Mais la dernière, en suçant le sein de sa mère adoptive, a permis aussi que se fasse, sans trop de heurts, le passage d'une parenté à l'autre. En aurait-il été autrement si la fratrie avait été sexuellement hétérogène? L'ambivalence fondamentale qui existe dans toute relation fraternelle a-t-elle été ici atténuée du fait que le groupe des adoptés n'était composé que de filles?

On peut aussi se poser la question de savoir si l'âge des fillettes au moment de l'adoption a été déterminant. L’aînée était suffisamment âgée pour pouvoir tenir auprès des autres une place tutélaire, sorte de gardienne des origines natales. La benjamine, encore très jeune, a pu prendre celle d'intégratrice au groupe adoptant. La puînée a sans doute désamorcé la rivalité qui aurait pu s'instaurer entre la première et la dernière. Chacune a joué son rôle de relayeur entre l'une et l'autre culture, entre l'une et l'autre parenté, entre les sœurs de l'une à l'autre. Parce qu'elles avaient l'âge requis pour tenir ces positions, l'adoption a $\mathrm{pu}$, ici, prendre place harmonieusement. Les fillettes ont acquis une nouvelle identité et sont entrées, à part entière, dans de nouveaux rapports de parenté tout en gardant mémoire de leur ancienne parenté.

De plus, le fait de tout connaître de leurs origines natales et de leur histoire adoptive, de savoir que du côté de leurs adoptants cette dernière bénéficie d'une image positive voire valorisante, tous ces traits ont, certainement, constitué une part non négligeable dans la réussite de cette famille adoptive.

Enfin, faut-il insister, à propos de cette adoption, sur le fait qu'elle intervient dans une société où la dimension consanguine, verticale ou horizontale, est inscrite frontalement dans la culture parentale. La force de la 
consanguinité s'est concrétisée, nous l'avons noté plus haut, par la parfaite harmonie présentée par la parenté des parents adoptifs et la germanité des adoptés. Les uns et les autres formaient bloc, groupe; ils se sont mutuellement épaulés, reconnus. Filiation et germanité se sont conjuguées pour faire aboutir cette adoption. Tout en les élevant, les éduquant, leur donnant une nouvelle identité, les parents adoptifs ont permis, à travers cette germanité, que quelque chose se transmette harmonieusement entre filiation d'origine et filiation acquise. L'histoire générationnelle et familiale des enfants n'a pas été remise en cause. Les sœurs sont restées " elles" tout en devenant "autres». Exactement comme dans leur culture d'adoption, l'étranger reste à jamais un horsain, mais en acquérant l'identité locale, en adoptant les mours du pays, en s'inscrivant dans une parenté, il peut y être admis, affilié : ces jeunes Coréennes nommées D., pourvues de prénoms chrétiens, installées ici, sont devenues normandes.

Il faut toutefois nous interroger sur la cohérence globale que revêt notre observation et sur le bien-fondé de nos procédés de description. La présentation très factuelle du cas de ces sœurs coréennes devenues normandes soulève les problèmes de la qualité de sa restitution et celui de son exemplarité. On doit, à l'un et l'autre niveaux, s'enquérir sur le caractère représentatif et comparable de son observation.

Comment décrire sans altérer ni biaiser un univers aussi intime, aussi personnel, aussi secret, que cette entité adoptive. Certes, j'ai eu accès à certaines informations, mais par le seul truchement des adoptants. Dès lors, n'ai-je pas été influencée par leurs commentaires? J'ai observé leurs lieux de vie; je me suis entretenue avec leurs relations amicales ou familiales. Mais ai-je tout restitué de mes sources? Il est difficile de savoir si l'on a tout inventorié et si l'analyse proposée reflète bien l'expérience vécue ou se fonde sur elle. Qu'apportons-nous de plus que d'autres recherches issues d'autres disciplines?

L'intérêt théorique de ces analyses ethnographiques de terrain est de permettre une réflexion approfondie sur les modes de fabrication, en matière d'adoption, de l'identité familiale et sociale, en faisant jouer toutes les composantes de la parenté locale, ascendante et collatérale, biologique et sociale, mais en tenant compte aussi du contexte affectif et sociologique qui préside à cette élaboration.

Peut-on et doit-on, à partir de là, généraliser ? En la matière, les données quantifiées ne sont guère plus probantes ou concluantes, mais on ne saurait aussi attendre aucune généralité par l'addition de cas particuliers. 
"Une adoption ne fait pas l'autre», assure Monsieur D. Sommes-nous alors réduits, pour rendre compte de ce sujet, à ne proposer qu'une série d'énoncés singuliers sans liens entre eux?

Nous croyons, cependant, que quelques traits saillants émergent de cette présentation de cas. D'abord, nous avons voulu montrer comment les données sur l'adoption internationale puisent logique et permanence dans le modèle même de la parenté locale. Ensuite, nous avons insisté sur les façons dont un agencement de faits biologiques et symboliques concoctent une figuration familière et familiale de la parenté. En d'autres termes encore, nous avons tenté de montrer comment le biologique (ici la germanité ) garde toute sa force et sa place dans les cas où il faut "faire famille» sans qu'aucun lien de sang ne rassemble ceux qui vont vivre ensemble en tant que parents. De même, avons-nous pointé les processus de "dénaturalisation" qui se mettent en place, alors que, par ailleurs, d'autres procédés visent à garder quelque chose de l'origine culturelle des adoptées. C'est de ce jeu de miroirs entre deux mondes dont il est question ici, et il éclaire de façon exemplaire certains paramètres de la production d'une identité filiale.

C'est bien de cet univers touffu où se fabrique la parenté que nous avons voulu rendre accessible au travers de cette présentation.

École des hautes études en sciences sociales Laboratoire d'anthropologie sociale, Paris zonabend@ehess.fr

MOTS CLÉS/KEYWORDS : adoption internationale/ international adoptions - nouvelles parentés/ new forms of kinship - mémoire des origines/ the memory of one's origins - construction de la parentél construction of kinship - filiation/descent - germanité/siblings. 
Belleau, Hélène

2000 "L'usage des photographies de famille dans l'élaboration de l'identité des enfants adoptés à l'étranger ", in Agnès Fine $\&$ Claire Neirinck, eds, Parents de sang, parents adoptifs: approches juridiques et anthropologiques de l'adoption (France, Europe, USA, Canada). Paris, L.G.D.J. : 311-323.

\section{Collard, Chantal}

1996 "Nouer, dénouer le cordon ombilical : illégitimité et adoption au Québec", Gradhiva 19: 53-62.

2005 «Triste terrain de jeu : à propos de l'adoption internationale ", Gradhiva 1, n.s. : 209-223.

2007 «Les nouvelles mises en forme de la filiation et de la mémoire généalogique au Québec " (à paraître).

\section{Crine, Anne-Marie \& Sylvia Nabinger}

1991 «Le roman familial des fratries dans l'adoption internationale ", Dialogue 114 : 35-41.

\section{De Heusch, Luc}

2002 "L'ennemi ethnique ", Raisons politiques: études de pensée politique 5, n.s. : L'Ennemi: 53-67.

\section{Delaisi de Parseval, Geneviève}

1981 La Part du père. Paris, Le Seuil.

\section{Fine, Agnès, ed.}

1998 Adoptions: ethnologie des parentés choisies. Paris, Éd. de la Maison des sciences de l'homme.

\section{Lechanteur, Fernand}

1974 «Les deux populations du département de la Manche", Parlers et traditions populaires de Normandie 6 : 81-101.
Martial, Agnès

1998 «Partages et fraternités dans les familles recomposées ", in Agnès Fine, ed., Adoptions : ethnologie des parentés choisies. Paris, Éd. de la Maison des sciences de l'homme : 206-244.

Monestier, Barbara

2005 Dis merci. Paris, Anne Carrière.

Ouellette, Françoise-Romaine

2004 "Adopter, c'est donner", in Isabelle Leblic, ed., De l'adoption: des pratiques de filiation différentes. Clermont-Ferrand,

Presses universitaires Blaise-Pascal:

269-291.

Pisier, Évelyne

2004 Une question d'âge. Paris, Stock.

Rouil, Jacques

2005 Les Rustres. [S. 1.], Cheminements.

Sagnes, Sylvie

2000 "La revanche élective : filiation adoptive et "origines" en France et aux ÉtatsUnis ", in Agnès Fine \& Claire Neirinck, eds, Parents de sang, parents adoptifs:

approches juridiques et anthropologiques de l'adoption (France, Europe, USA, Canada). Paris, L.G.D.J. : 169-183.

\section{Testart, Alain}

1999 «Ce que merci veut dire : esclaves et gens de rien sur la Côte Nord-Ouest américaine ", L'Homme 152 : 9-28.

\section{Zonabend, Françoise}

1991 «Mes frères, mes époux : fonctionnement de la parenté et figures d'alliance en Basse-Normandie ", in Françoise HéritierAugé \& Élisabeth Copet-Rougier, eds, Les Complexités de l'alliance: les systèmes complexes d'alliance matrimoniale. Paris, Éd. des Archives contemporaines : II, 206-226. 2004 Mours normandes. Paris, C. Bourgois. 
Françoise Zonabend, Adopter des sœurs. Construction de la parenté et mémoire des origines. - À partir du cas de l'adoption de trois sœurs coréennes par un couple originaire de la région du nord Cotentin (Manche, France), nous avons cherché à accéder à l'ensemble des éléments qui caractérisent notre système de parenté tant dans ses dimensions verticales qu'horizontales, que dans son expression restreinte ou étendue. L'étude de cet exemple permet d'appréhender et d'analyser la complexité des relations familiales que se tissent ou se rompent dans les groupes de parentés preneurs ou donneurs d'enfants, dès lors qu'on a recours à l'adoption lointaine pour se fabriquer des descendants ou qu'on doit, pour diverses raisons, abandonner ces derniers à des parents adoptifs. Situé dans ce contexte normand, il était loisible de s'interroger, de plus, sur la part que tient l'environnement local sur les façons de faire actuelles en matière de construction de la parenté et de la mise en place de mémoires partagées. Enfin, il est important de souligner le poids que pèse, dans le processus adoptif, la germanité prise, ici, dans toutes ses expressions de sexe, d'âge, de biologique et de social.
Françoise Zonabend, Adopting Sisters: Constructing Kinship and Remembering One's Origins. - The case of three Korean sisters adopted by a couple from northern Cotentin (Manche, France) has been used to grasp all the elements that characterize our kinship system in both its vertical and horizontal dimensions as well as in its restricted and extended forms. We can thus understand and analyze the complexity of the family ties, made or broken, in the groups giving or receiving children, when adoption from a far-off place «makes » descendants or when, for various reasons, offspring are abandoned to adoptive parents. In the Normand context, it was possible to inquire into the local environment's impact on the current ways of constructing kinship and sharing memories. Attention is drawn to the weight carried in the adoption process by sibling ship in anything related to sex and age, whether biological or social. This example serves to show how biological factors (in this case, full blood ties) still have a strong impact when a family is to be made without any blood ties relating the persons who will be living together. 